\title{
Effect of tomography resolution on the calculated microscopic properties of porous materials: Comparison of sandstone and carbonate rocks
}

\author{
R. Gooya, ${ }^{\text {a) }}$ S. Bruns, D. Müter, A. Moaddel, R. P. Harti, ${ }^{\text {b) }}$ S. L. S. Stipp, and H. O. Sørensen \\ Nano-Science Center, Department of Chemistry, University of Copenhagen, Universitetsparken 5 , \\ 2100 Copenhagen $\emptyset$, Denmark
}

(Received 15 June 2016; accepted 25 August 2016; published online 9 September 2016)

\begin{abstract}
$\mathrm{X}$-ray computed tomography is useful for providing insight into the internal structure of porous materials. Extracting reliable quantitative information is difficult because the derived properties rely heavily on data resolution, i.e., very different values emerge, depending on the relationship between size of the features in the sample and the resolution of the 3D tomograms. Here, we present a method for testing if resolution is sufficient for determining reliable petrophysical parameters, i.e., with low levels of uncertainty. We derived the physical properties of sandstone and carbonate rocks over a range of voxel dimensions by computationally reducing raw data resolution in our high resolution images. Lower resolution decreases the calculated surface area for all samples and increases the derived permeability for sandstone. The permeability vs change in resolution was not monotonic for carbonates. The differences in trends for the two rock types result from different pore sizes and pore size distributions. Published by AIP Publishing. [http://dx.doi.org/10.1063/1.4962389]
\end{abstract}

Sandstone and carbonate rocks host a significant portion of the global hydrocarbon resources and huge quantities of groundwater. Understanding how the petrophysical properties of the rock, e.g., porosity and permeability, influence fluid flow is therefore essential for predicting production of oil and water and also the migration of contaminants in soil, sediments, and rocks. ${ }^{1}$ Permeability is usually determined by core plug testing but with recent advances in design, X-ray microtomography is now able to deliver 3D images with resolution high enough to distinguish features in even very fine grained materials such as chalk. ${ }^{2-4}$ This opens an opportunity to study how pore morphology, pore size distribution, and connectivity ${ }^{5}$ control the microscopic properties of the rock and fluid flow through the pore system.

One factor that has a profound effect on the simulation results is the image resolution. ${ }^{6-8}$ Relatively low resolution can be sufficient for rocks with large pore throats, e.g., sandstones ${ }^{9}$ and coarse grained carbonate rocks, but in tight carbonates, particles and pores are nanometre scale. ${ }^{10}$ Consequently, image resolution has a considerable influence on the derived petrophysical parameters. The relationship between resolution and parameter uncertainty has been investigated for samples of chalk $^{11}$ and that study demonstrated that an increase in the resolution caused an increase in specific surface area and a decrease in porosity and permeability.

In this work, we investigated the impact of X-ray microtomography image resolution on the microscopic properties of sandstones and carbonate rocks. Using images with $0.65 \mu \mathrm{m} /$ voxel resolution, we decreased the resolution by scaling, first in small steps to 0.8 and $1.0 \mu \mathrm{m} /$ voxel and then in larger steps to 1.5 and $2.0 \mu \mathrm{m} /$ voxel. This resolution is in the range that is

\footnotetext{
a) Author to whom correspondence should be addressed. Electronic mail: gooya@nano.ku.dk

b) Current address: Paul Scherrer Institute, CH-5232 Villigen-PSI, Switzerland.
}

common for high resolution X-ray microtomography imaging, used for rock samples. ${ }^{12,13}$

$\mathrm{X}$-ray microtomography data were collected for two carbonate rocks (C1: Dolomite 1 and C2: Dolomite 4) and three sandstones (S1: Sandstone BAii, S2: Sandstone BBii, and S3: Sandstone Core C cleaned), at the TOMCAT beamline at the Swiss Light Source (SLS) at the Paul Scherrer Institute (PSI), Villigen, Switzerland. We recorded 1501 radiographic projections while the sample was rotated through $180^{\circ}$. Exposure time was $250 \mathrm{~ms}$ per projection. The projections were corrected for detector dark current and flood field and the tomograms were reconstructed using the GridRec software ${ }^{14}$ with the gridrec reconstruction algorithm with a voxel size of $650 \mathrm{~nm}$. Further details on the data collection and reconstruction methods can be found elsewhere. ${ }^{15}$

The $0.65 \mu \mathrm{m}$ voxel dimension greyscale X-ray tomography images were used as a starting point to emulate the effects of coarser resolution. These data were corrected for high frequency noise using in house developed denoising software ${ }^{16}$ to provide a clean, segmentable signal (Figure 1). We divided the scaling of the voxel sizes into two steps because simple rebinning of the data would only imitate undersampling of the available image information. We limited the bandwidth of the signal with a Gaussian low pass filter and then adjusted the sampling rate to match the initial ratio of voxel size and physical image resolution. For this, it was assumed that the voxel dimension of $0.65 \mu \mathrm{m}$ in the available data coincided with the physical image resolution, given by the full width at half maximum of the point spread function (PSF) that we assumed to be of Gaussian form. With additive variance, the standard deviation of an appropriate Gaussian filter kernel $\left(\sigma_{\text {filter }}\right)$ that reduces the image resolution by the desired factor, $\mathrm{f}$, is then simply given by

$$
\sigma_{\text {filter }}^{2}=\sigma_{\text {lowRes }}^{2}-\sigma_{\text {tomo }}^{2}=F W H M^{2} \cdot \frac{f^{2}-1}{8 \cdot \ln 2},
$$


(a)

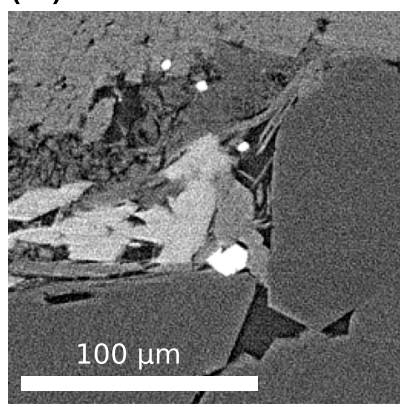

(d)

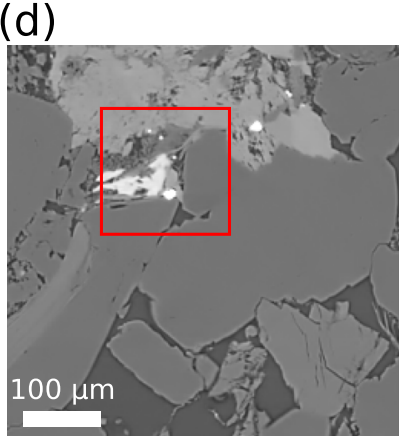

(b)

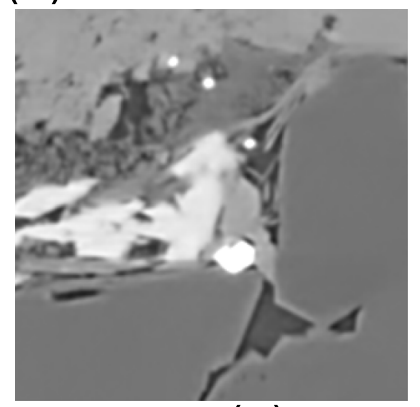

(e)

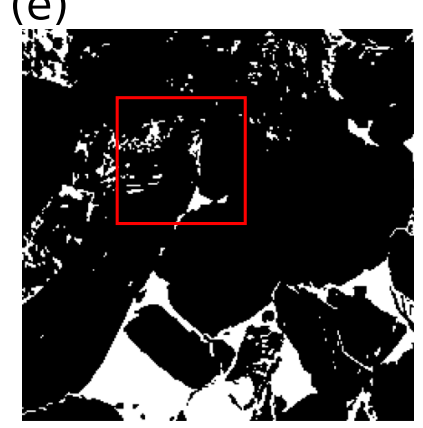

(c)

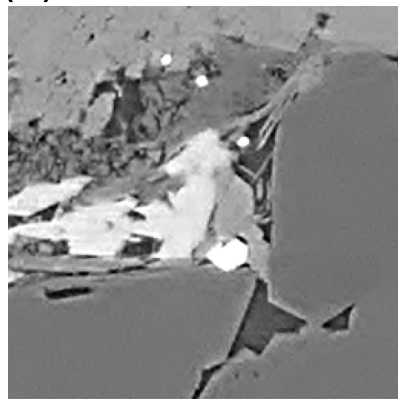

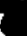

FIG. 1. The image processing steps. A $256 \times 256$ pixel slice through a 3D image from sandstone. The reconstructed tomography data before (a) and after (b) nonlocal means based denoising at $0.65 \mu \mathrm{m}$ resolution. The image was low pass filtered to an effective resolution of $2.0 \mu \mathrm{m}$ (c) and transferred to a grid of $2.0 \mu \mathrm{m}$ voxel resolution (d), then binarized by multilevel Otsu thresholding (e). The red square indicates the location of images (a)-(c) in the broader scale images. where $\sigma_{\text {tomo }}$ and $\sigma_{\text {lowRes }}$ represent the standard deviation of the PSF before and after low pass filtering and FWHM represents the full width at half maximum of the PSF before low pass filtering. For each dataset, down sampled versions were produced, with resolutions of $0.8,1.0,1.5$, and $2.0 \mu \mathrm{m}$. These images were oversampled on the original grid. They were transferred to a lower resolution grid by linear interpolation, such that $F W H M$ and voxel dimension remained identical. Finally, all images were segmented using slice-by-slice multilevel Otsu thresholding. ${ }^{17}$ The multilevel approach was essential to avoid minerals of varying attenuation biasing the segmentation results.

Once the data were segmented, the same volumes, i.e., $130^{3} \mu \mathrm{m}^{3}$, at resolutions of $0.65\left(200^{3}\right.$ voxels $), 0.8\left(161^{3}\right.$ voxels), 1.0 (131 $1^{3}$ voxels), 1.5 (98 voxels), and $2.0\left(65^{3}\right.$ voxels) $\mu \mathrm{m} /$ voxel were selected. The porosity, $\varphi$, surface area, $A$, and pore dimension could be derived from the binarized images. The surface area was determined by isosurfaces, using the marching cube algorithm ${ }^{18}$ and porosity was assumed to be the ratio of pore voxels to the total number of voxels (i.e., the volume of the voids). To find the pore size distribution in 3D tomographic images, we used a watershed algorithm on a distance map of the 3D structure to isolate pores and find the pore network of the sample. For each pore, the radius of the largest sphere to fit inside the pore was considered to be the pore size.

Incompressible, viscous, and Newtonian flow equations were solved using a finite volume method. ${ }^{15}$ Continuity and momentum equations are

$$
\Delta p=\mu \Delta^{2} \boldsymbol{v}
$$

and

$$
\Delta \cdot \boldsymbol{v}=0,
$$

where $\mu$ represents viscosity of the fluid, $\rho$ represents density, $\Delta p$ the pressure gradient, and $\boldsymbol{v}$ velocity. The semi-implicit pressure based (SIMPLE) approach was implemented in OpenFOAM libraries ${ }^{19}$ for coupling of velocity and pressure. No-slip boundary conditions were applied on the walls for velocity and a pressure gradient of $1 \mathrm{~Pa}$ was applied on the $x$ direction. The water permeability of subvolumes was determined using the Darcy equation. Further details on the simulations can be found elsewhere. ${ }^{15}$

Figure 2(a) shows 2D slices of S1 and C2 at three levels of resolution (examples of the 3D volumes are presented in supplementary material $\mathrm{S} 1$, a sandstone, includes a range of pore sizes, whereas the pores of the carbonate sample, $\mathrm{C} 2$, are small; for the carbonate samples in general, most pore radii are $<10 \mu \mathrm{m}$ (Figure 2(b)), whereas in the sandstone samples, pore radius is generally $>5 \mu \mathrm{m}$. The average pore dimensions, $\mathrm{C} 1: 2.23 \mu \mathrm{m}, \mathrm{C} 2: 1.70 \mu \mathrm{m}, \mathrm{S} 1: 1.83 \mu \mathrm{m}, \mathrm{S} 2: 1.73 \mu \mathrm{m}$, and S3: $5.07 \mu \mathrm{m}$, are not so different but the volume ratio occupied by different sizes is quite different (Figure 2(b)).

The effect of changing image resolution on the derived parameters can be categorized into three regimes. Square 1 in Figure 2(a) shows an example of the first regime, where two pores that are visibly not connected at the highest resolution, appear to be connected at lower resolution and some connected pores appear unconnected. False connection was most common in volumes of high porosity and pore disconnection, in areas of lower porosity. Errors in pore connectivity of both types occurred in volumes with narrow pore throats. The second regime, for example, in Square 2, is where isolated or somewhat isolated solid material disappears, making the pores appear to be smoother. The third regime, represented by Square 3, shows how narrow pores in the solid completely disappear at lower resolution. Also, as resolution decreases, pores become more regular, rectangular, or square. From the pore size distributions of the samples and the observed effects of changing resolution, we can categorize the effect of resolution decrease into these three regimes.

Figure 3 schematically shows the type of effect on the derived parameters in relation to pore size for sandstone and 
(a)

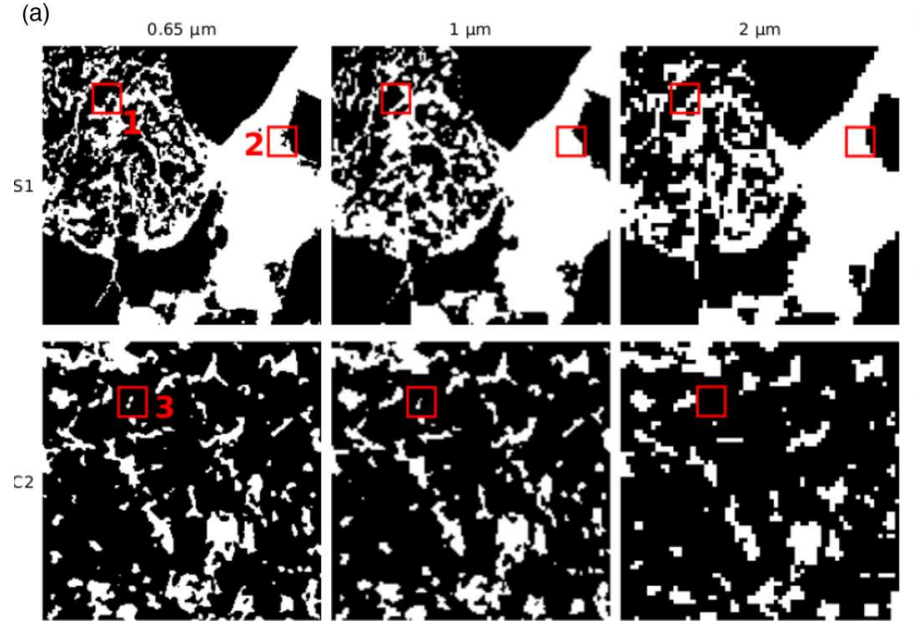

(b)

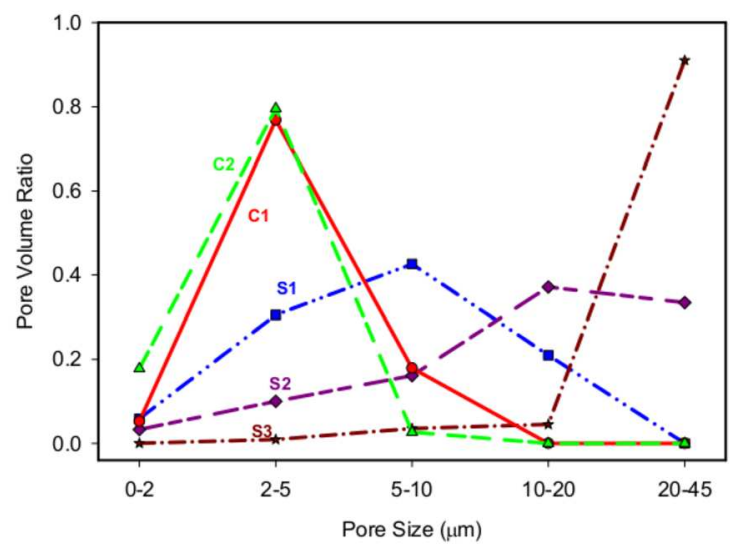

FIG. 2. (a) 2D slices of sandstone (S1) and carbonate (C2) rocks at a range of resolution; in the first column are tomography data with $200^{3}$ voxels, the second column is treated data from the same volume with $131^{3}$ voxels, and the last column is treated data with $65^{3}$ voxels. White represents pores and black the solid. (b) Pore size distributions (PSDs) for all of the samples at $0.65 \mu \mathrm{m} /$ voxel size, pore volume ratio is the ratio of pore volume to the total volume of the sample.

carbonate samples. Tomograms from sandstones are mostly affected by smoothing of the main pores, i.e., Regime 2, and samples with small pores are affected most by disappearance, Regime 3, and connection or disconnection of pores, Regime 1. Figure 3 presents the relationships qualitatively and on a relative scale, so resolution and pore size distribution must be considered together. For example, if we consider a sample where most of its pore volume is occupied by pores with $\sim 2 \mu \mathrm{m}$ radii, we would need to know the tomography resolution to predict which part of Figure 3 would describe our sample. If we consider tomograms with $2 \mu \mathrm{m}$ resolution, the resolution and the dominant pore size are similar, so the effect of decreased resolution follows Regime 3, as described at the bottom part of Figure 3, i.e., features disappear. In microtomography, where optical resolution is $\sim 1 \mu \mathrm{m}$, sandstone samples with large pores fall in the upper part of Figure 3 and

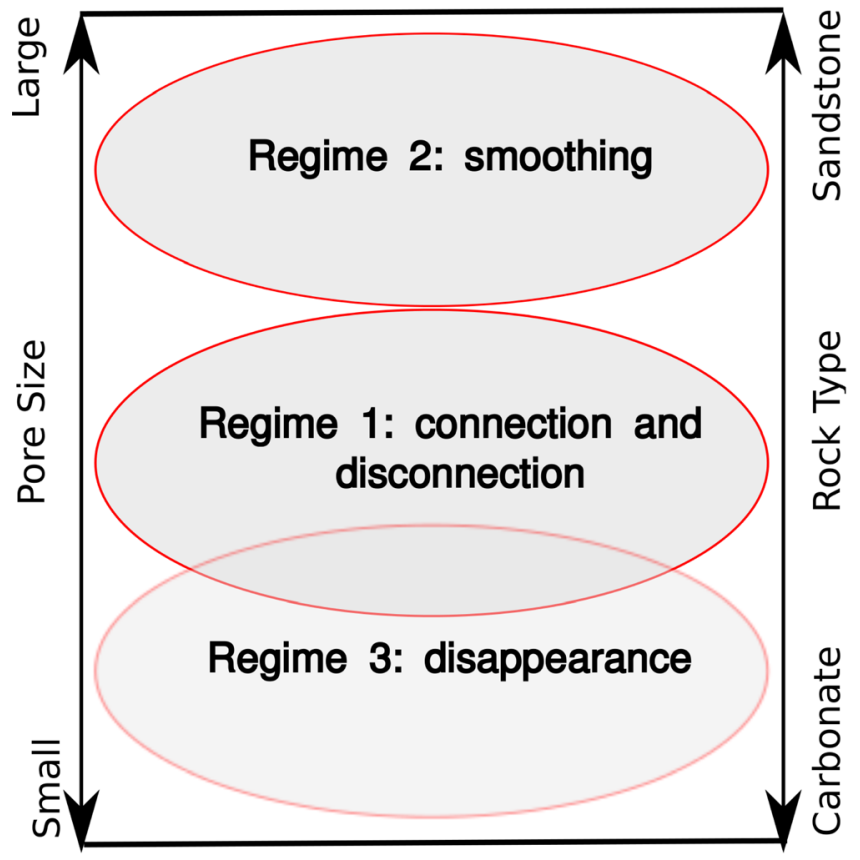

FIG. 3. The dependence of the loss of resolution on pore size distribution for sandstone and carbonate rocks. carbonate rocks in the lower part because their pore size is $\sim 1 \mu \mathrm{m}$.

Figure 4 shows porosity, surface area, and permeability ratio for the carbonate and sandstone samples as a function of image resolution. As resolution decreases, the porosity determined from the carbonate samples decreases but porosity for the sandstone samples is not significantly affected. This makes sense because the pores are larger than the voxel dimension. Porosity decreases in the carbonate samples because the pores are narrow compared with the voxel size, so features disappear as the voxel size increases. The porosity of samples S1 and S2 remains constant because the increase in the porosity in large pores compensates for the decrease in the porosity in small pores. Sample S3 porosity increased slightly because it mainly includes large pores where the surfaces are generally smoothed, which is also why the surface area is slightly reduced, Figure 4(a). We also found that there is higher uncertainty in segmentation error for porosity at lower resolution using numerical erosion and dilation of the data. ${ }^{20}$ Details of the segmentation error are presented in supplementary material. Compared with sandstone, the surface area for carbonate rocks is higher because there are many small pores. ${ }^{21}$ Surface area decreases as the resolution decreases because small features disappear.

The dependence of permeability on voxel dimension is more complex because of the influence of more than one effect. The data derived from the three sandstone samples had trends that were similar. Permeability increased with voxel size because the effect of decreased resolution is smoothing of the pores, so fluid flows with less resistance. The fluctuations for the carbonate samples are more profound. Disconnection of pores occurs for Sample C1 when the voxel dimension increases from 0.65 to $1.0 \mu \mathrm{m} / \mathrm{voxel}$ and as it increases further, reconnecting pores dominate so initially, permeability decreases, then increases (Figure 4(c)). Permeability change for $\mathrm{C} 2$ is opposite, probably because the influence of added connections and loss of connections is slightly out of balance. This is a demonstration that as the voxel dimension approaches or becomes greater than the dimension of the features, uncertainty increases. Examples of the pressure field are presented in supplementary material. 

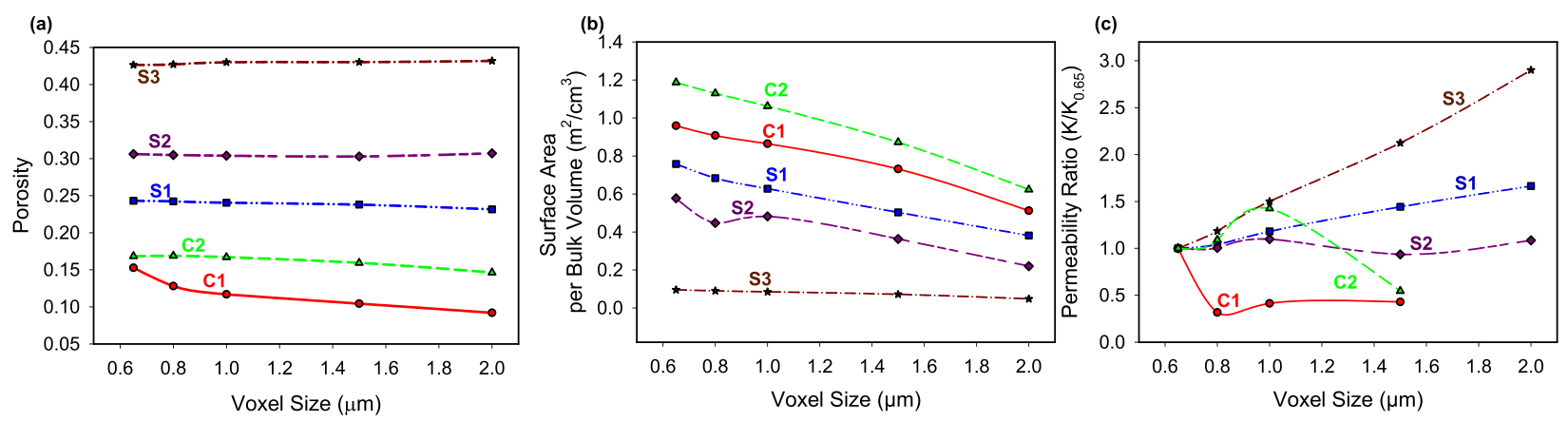

FIG. 4. (a) Porosity, (b) surface area, and (c) permeability ratio as a function of voxel dimension for the five samples. The permeability ratio is defined as K divided by the $\mathrm{K}$ at $0.65 \mu \mathrm{m} /$ voxel dimension. The permeability plots for the carbonate samples have only been shown for voxel dimensions up to $1.5 \mu \mathrm{m}$ because at lower resolution, pore disappearance leads to no consistent connectivity through the $130^{3} \mu \mathrm{m}^{3}$ volume.

$\mathrm{X}$-ray tomography provides insight into the physical properties of porous materials, allowing us to gain understanding about the influence of internal structure on macroscopic behavior. One criterion, however, for deriving parameters with low uncertainty is that the resolution of the 3D images must be good enough. This is particularly a challenge for natural porous materials because they are usually quite heterogeneous, so there is a complex relationship between resolution and the effects that low resolution causes. In rare cases, where multiple images of the same sample are acquired at different scales, it is possible to determine if the obtained resolution is sufficient ${ }^{11}$ but most often it is only possible to image a sample at one resolution.

By computationally decreasing the amount of data in a dataset, thereby reducing the resolution, we can sequentially follow the influence of the resolution on the extracted parameters. The relationship between the properties and the resolution provides an indication of whether the initial data set has sufficient resolution to reliably predict the extracted properties. We tested the method on two different rock types, sandstones and dolomitic limestone. We found that the effect of changing the resolution produced a variety of effects that depended on the size of the features in the porous medium relative to the voxel size. Smoothing of the pores was the dominant effect for the large pores in sandstone and false connection or disconnection, as well as disappearing pores, dominated for the fine grained carbonate rocks. We can conclude that when the size of the voxels is much smaller than most of the pores, permeability increases with increasing voxel size and system behavior is predictable. However, when the voxel size is larger or on the same order as the pore size, the uncertainty introduced by loss of resolution is unpredictable.

These results can be used to provide insight into how calculated properties of porous materials would be affected by insufficient resolution. The main variable is pore size, which is linked to the rock type. As expected, voxel size on the order of $1.0 \mu \mathrm{m}$ is enough for reasonable prediction of sandstone properties if clay is a minor component of the rock. This work shows that some parameters can be predicted for the carbonate rocks but lower resolution misses their narrow pores and pore throats, precluding the possibility of reliable estimates of permeability.
See supplementary material for the 3D volumes of samples, pressure fields, and segmentation error.

We acknowledge funding from the Innovation Fund Denmark and Maersk Oil and Gas A/S, for the $\mathrm{P}^{3}$ Project and Innovation Fund Denmark through the CINEMA Project. Beamtime travel costs were founded by Danish Council for Independent Research [via DANSCATT]. We thank D. Haberthür and K. N. Dalby for technical support and Finn Engstrøm for useful discussions and providing the dolomite samples. R.G. and A.M. acknowledge a fellowship from the $\mathrm{CO}_{2}$ REACT Project of the European Commission 7th Framework Programme, No. 317235.

${ }^{1}$ F. Luo, J. Hou, Z. Li, S. Bing, and S. Wang, Energy Fuels 25, 4414 (2011). ${ }^{2}$ F. Marone, C. Hintermüller, S. McDonald, R. Abela, G. Mikuljan, A. Isenegger, and M. Stampanoni, "X-ray tomographic microscopy at TOMCAT," Proc. SPIE 7078, 707822 (2008).

${ }^{3}$ M. J. Blunt, Curr. Opin. Colloid Interface Sci. 6, 197 (2001).

${ }^{4}$ D. Müter, H. O. Sørensen, H. Bock, and S. L. S. Stipp, J. Phys. Chem. C 119, 10329 (2015).

${ }^{5}$ V. Cnudde and M. N. Boone, Earth-Sci. Rev. 123, 1-17 (2013).

${ }^{6}$ H. Yoon and T. A. Dewers, Geophys. Res. Lett. 40, 4294, doi:10.1002/ grl.50803 (2013).

${ }^{7}$ V. Hebert, C. Garing, L. Luquot, P. A. Pezard, and P. Gouze, Geol. Soc., London, Spec. Publ. 406, 61 (2015).

${ }^{8}$ F. Jinuntuya, R. Chen, H. Ostadi, K. Jiang, Y. Gao, and X. Zhang, ECS Trans. 48, 93 (2014).

${ }^{9}$ S. Peng, F. Marone, and S. Dultz, J. Hydrol. 510, 403 (2014).

${ }^{10}$ Q. Hu, R. P. Ewing, and S. Dultz, J. Contam. Hydrol. 133, 76 (2012).

${ }^{11}$ D. Müter, H. O. Sørensen, D. Jha, R. Harti, K. N. Dalby, H. Suhonen, R. Feidenhans, F. Engstrøm, and S. L. S. Stipp, Appl. Phys. Lett. 105, 043108 (2014).

${ }^{12}$ C. Soulaine, F. Gjetvaj, C. Garing, S. Roman, A. Russian, P. Gouze, and H. A. Tchelepi, Transp. Porous Media 113, 227 (2016).

${ }^{13}$ C. Noiriel, Rev. Mineral. Geochem. 80, 247 (2015).

${ }^{14}$ F. Marone and M. Stampanoni, J. Synchrotron Radiat. 19, 1029 (2012).

${ }^{15}$ R. Gooya, H. O. Sørensen, D. Müter, Y. Kanzaki, R. P. Harti, S. S. Hakim, K. N. Dalby, and S. L. S. Stipp, "Pore Scale Analysis of Carbonate Rocks Using High Resolution X-ray Microtomography Images," Geophys. J. Int. (to be published).

${ }^{16}$ S. Bruns, S. L. S. Stipp, and H. O. Sørensen, "Looking for the signal: a guide to noise and artefact removal in X-ray tomography reconstructions of chalk" (unpublished).

${ }^{17}$ N. Otsu, Automatica 11, 23 (1975).

${ }^{18}$ W. E. Lorensen and H. E. Cline, SIGGRAPH Comput. Graph. 21(4), 163-169 (1987).

${ }^{19}$ See www.openfoam.com for OpenCFD, 2013.

${ }^{20}$ F. Fusseis, C. Schrank, J. Liu, A. Karrech, S. Llana-Fúnez, X. Xiao, and K. Regenauer-Lieb, Solid Earth 3, 71 (2012).

${ }^{21}$ J. H. Schön, Physical Properties of Rocks: Fundamentals and Principles of Petrophysics (Elsevier, 2015), Vol. 65. 